\title{
Archi-intelligibilité johannique dans la phénoménologie de Michel Henry
}

\author{
Une réception phénoménologique radicale $d u$ \\ prologue de Jean
}

\author{
Rolf Kühn (Freiburg im Breisgau) \\ (traduit de l'allemand par Lukas Held)
}

Si la vérité du christianisme est la Vie divine elle-même dans toute sa plénitude, alors la révélation faite par Dieu aux hommes ne peut consister qu'en une autorévélation se donnant sans réserve. Cette réponse donnée par la phénoménologie matérielle fondée par Michel Henry, signifie alors pour la christologie que le « Fils » ou le «Verbe » doivent être compris comme le premier-vivant dans la mesure où il rend possible l'auto-révélation divine comme procès de vie intra-trinitaire. Avant d'examiner le prologue de Jean dans le sens de la phénoménologie matérielle, tel qu'il a été exposé dans C'est moi la vérité, il sera nécessaire de donner quelques renseignements généraux concernant les prérequis phénoménologiques qui y sont liés.

\section{1/ Auto-révélation de Dieu et vérité du christianisme}

Les écrits du Nouveau Testament laissent entrevoir que le christianisme associe avant tout - et donc par principe - la réalité de la révélation en tant qu' «autorévélation divine » à la vie. Il n'est plus possible qu'il s'y agisse de simples phrases, symboles ou images, mais il y est question de l'absoluité phénoménologique de notre propre vie: sans vie, nous n'existerions pas, nous ne percevrions pas, nous serions incapable d'effectuer la moindre action conditionnée par notre incarnation vivante. Depuis la fondation de la phénoménologie par Edmund Husserl, la vie subjective en tant que "présent vivant» a été considérée comme point de départ de toute manifestation, et ce jusqu'à ce que cette approche ait été radicalisée par Michel Henry, ce dernier ayant montré que la vie phénoménologique absolue reste essentiellement invisible et ne peut dont pas être représentée par une connaissance, intuition ou perception quelconque. En effet, la vie en tant que condition concédée ou donnée est non seulement présupposée dans l'exécution consciente ou inconsciente de chaque acte, mais aussi dans notre épreuve et notre sensation des choses. ${ }^{1}$ Cette condition tout aussi nécessaire qu'assurée en elle-même d'un point de vue aprio-

\footnotetext{
${ }^{1}$ Pour une confrontation avec la phénoménologie classique, cf. M. Henry, Incarnation. Une philosophie de la chair, Paris, Seuil, 2000, p. 35 sqq.
} 
rique ou transcendantal, Henry l'appelle « auto-affection » - en accord terminologique avec la tradition philosophique depuis Descartes et Kant jusqu'à Maine de Biran et Fichte - sans adopter pour autant les consignes rationalistes ou idéalistes de ce terme.

Ce mode de la vie qui nous affecte constituant notre origine absolue dans un sens radical ou matériel (d'un point de vue phénoménologique) sans que nous y ayons un accès réflexif (puisqu'il serait toujours en retard par rapport à la vie), il devient évident qu'il existe un lien immédiat entre la vie divine et notre vie. Le Christ est ce lien (religio) entre Dieu et nous, le Christ comme ce premier-vivant que nous avons déjà mentionné et que Henry désigne, dans le cinquième chapitre capital de C'est moi la Vérité, comme l'archi-fils. L'adoption divine dans le processus d'autogénération éternellement vivante de Dieu le Père constitue le moment de révélation décisif en tant qu'auto-détermination concrète de ce processus intradivin comme étant un processus d'engendrement et de réceptivité : le «Père» se comprend comme la vie principielle elle-même et cette auto-compréhension est en même temps son «Verbe» ou logos dans lequel s'exprime cette vie archi-originelle comme une vie toujours vivante afin de se rendre à sa propre origine dans une telle auto-compréhension sans aucun écart entre Père et Fils eu égard à leur essence divine et à la "réciprocité interne » y étant impliquée. Puisque la vie phénoménologique absolue interdit toute différence ou écart dans le sens d'une ek-stase, d'une transcendance ou d'un horizon, ces catégories ne peuvent être transposées à Dieu. Ainsi, l'approche phénoménologiquement matérielle de Henry est en accord immédiat avec la pensée de Maître Eckhart. ${ }^{1}$

Ce processus intradivin et autosuffisant implique en outre que le Dire de Dieu (logos, parole) et le «retour» aimant réalisé en réciprocité identique ou interne est partout et toujours la vie - et rien d'autre que la vie. Or, par le fait même que l'auto-expression de la vie comme compréhension de soi en Dieu contient, nous l'avons dit, sa propre réceptivité à travers laquelle elle s'exprime, elle comporte une « ipséité » unique qui devient — en tant qu'« archi-fils » - le principe de toute vie individuée. Ainsi, l'accès à la vie ou plutôt à notre «naissance transcendantale » en elle n'existe que grâce à la concrétion archi-christologique de la vie divine en son Verbe ou, comme le dit le prologue de Jean, le Christ, logos éternel qui était « dans le Père au commencement » est la vérité du monde en tant que vie pour tout vivant. Dans la mesure où notre propre «auto-rapport» dans le « Moi » ainsi que notre « hétéro-rapport» à l' «Autre» ne sont possibles qu'en tant que rapports vivants,

\footnotetext{
${ }^{1}$ Cf. M. Henry, L'essence de la manifestation, Paris, PUF 1963, § 39-40, 49-50 ; « Acheminement vers la question de Dieu : preuve de l'être ou épreuve de la vie », dans Phénoménologie de la vie, t. IV : Sur l'éthique de la religion, Paris, PUF, « Epiméthée », 2004, p. 67-80 ; R. Kühn et S. Laoureux (dir.), Meister Eckhart - Mystik und Erkenntnis des Lebens. Forschungsbeiträge der Lebensphänomenologie, Fribourg/Munich, Alber 2008, R. Kühn, "Ungeteilheit » - oder Mystik als Ab-Grund der Erfahrung. Ein radikal phänomenologisches Gespräch mit Meister Eckhart, LeydenBoston, Brill. 2012.
} 
cela implique que l'ipséité archi-christologique, c'est-à-dire la vie, est au fondement de toutes ces relations. Il nous est impossible de faire un pas vers l'autre ou vers nous-mêmes sans entrer en relation dans notre « chair » avec la «chair» du Christ. Nous ne sommes donc pas seulement «Fils de Dieu» mais plus précisément, comme le disait Saint Paul, « Fils du Fils », c'est-à-dire des vivants par la vie divine de l’Un, du « premier-né divin ». ${ }^{1}$

Henry présente la vérité du christianisme dans la lumière d'une «phénoménologie jusqu'à présent sans exemple et non-utilisée » qui ne relie plus l'essence humaine à la ratio ou à l'être mais à la vie qui est toujours et par principe une vie individuée. L'interprétation que fait la phénoménologie matérielle du prologue de Jean n'a donc pas seulement une importance pour la phénoménologie ou la philosophie de la religion. Elle réattribue un rayonnement puissant au Christianisme pour le futur face à la menace de la vie individuelle et sociale par les différentes théories d'une « objectivité » économico-technique. La liaison absolue du christianisme à l'essence de la vie absolue - Dieu - serait capable de redonner à l'humanité entière ainsi qu'à chaque individu une certaine dignité, indépendamment de tous les facteurs extérieurs tels que race, sexe, situation sociale, éducation, etc., qui n'aurait nul besoin de théorie, ou plus généralement de langage ou de raison, mais qui serait fondée immédiatement dans la facticité « charnelle » et vivante de chaque existence respective.

La priorité vitale de la liaison entre homme et Dieu telle qu'elle est décrite dans le prologue de Jean a également des conséquences pour une "éthique chrétienne », entendue comme une analyse transcendantale des rapports du christianisme au monde. Il en ressort en effet que la croyance chrétienne en la révélation ne peut jamais être une « fuite du monde », comme le pensaient déjà Hegel et un marxisme doctrinaire. C'est justement le commandement de l'amour du prochain qui renvoie à une pratique qui concerne, en tant que regeneratio de la vie, tous les besoins matériels ou spirituels de l'homme dans leur origine même. Autrement dit: l'éthique chrétienne n'est pas un impératif qui s'ajouterait de l'extérieur à la vie comme une norme qui lui serait étrangère et qui ne fournirait ni la motivation ni la force pour l'agir respectif. Michel Henry montre, à travers l'exemple des « œuvres de miséricorde » et de la confrontation entre Nouveau et Ancien Testament au sujet de la « loi ancienne », qu'une éthique du christianisme dans la perspective du prologue de Jean correspond exactement à l'ontologie phénoménologique de la vie qui fonde la « vérité du christianisme » - comme cette manière de retrouver au sein de la « seconde naissance » son origine en Dieu, et pour autant que celle-ci ait été oubliée de manière égoïque et en raison d'une « illusion transcendantale ». ${ }^{2}$

D'un point de vue de l'histoire des idées et des faits, il est remarquable que la philosophie moderne de Descartes à Heidegger, après la définition antique et médiévale

${ }^{1}$ Cf. M. Henry, C'est moi la vérité. Pour une philosophie du christianisme, Paris, Seuil, 1996, chap. 6-7: "L'homme en tant que "Fils de Dieu" / / "L'homme en tant que "Fils dans le Fils" ».

${ }^{2}$ Cf. ibid., chap. 9 : « La seconde naissance »; Incarnation, op. cit., $\S 45$ et 48. 
de l'être humain comme "être doué de raison» ou «créature finie», ait mis au centre de sa réflexion l'être-sujet intentionnel ou encore le caractère «souciant»du Dasein. Or, on n'a pas pris en compte le fait que toute accentuation subjective ou relation compréhensive à l'être présuppose une passivité originaire que Henry désigne par le terme de passibilité - en raison du lien affectif entre vie et chair. Ceci éclaire le fait que toute possibilité de la vie, que toute «potentialité » n'existe que grâce à une réception originaire passible qui, en tant que naissance auto-affective authentique dans la vie, ne connaît qu'un simple « Moi » à l'accusatif, comme cela a déjà été souligné par Emmanuel Lévinas. Ainsi, nous dépassons le reproche postmoderne selon lequel le concept de sujet n'appartiendrait qu'à une approche subjectiviste désuète sur l'arrière-fond métaphysique d'une pensée substantielle ontologico-théologique. ${ }^{1}$ Il s'agira bien plutôt d'analyser tous les concepts différentiels ou «altériologiques» forgés jusqu'à présent par la philosophie dans la mesure où ils n'utilisent le concept de vie que comme « opacité » ou « trace», comme le font par exemple Merleau-Ponty et Derrida, sans élucider cette « obscurité » ou « invisibilité » dans le sens de la phénoménologie henryenne, c'est-à-dire comme le mode de manifestation immanent de la vie dans sa passibilité affective comme pathos. ${ }^{2}$

En reliant le prologue de Jean avec les autres discours de Jésus sur lui-même qui parcourt tout son évangile, on remarque ainsi - ces discours étant en première personne -, un « égocentrisme » semble-t-il on ne peut plus démesuré, mais qui ne peut être compris que sur base de la connaissance du caractère unique de l'adoption du Christ en Dieu et par Dieu. Car l'être-moi n'exclut pas, d'un point de vue christologique, l'«obéissance», puisqu'elle correspond, dans les limites des procédés divins et de leur réciprocité interne, exactement à l'essence du Fils : être de manière passiblement réceptive le Verbe vivant de l'auto-révélation divine. Ceci n'est qu'un éclaircissement de la réalité interne de la vie divine qui possède son «affinité » à travers les modes de modalisation absolus de la vie phénoménologique absolue, affinité repérée par Henry lui-même entre l'évangile de Jean et la phénoménologie de la vie. ${ }^{3}$ En acceptant d'y voir l'une des plus grandes avancées de la pensée phénoménologique et de la philosophie de la religion, de par sa méthode et son conte$\mathrm{nu}^{4}$, il devient évident qu'il ne s'agit pas simplement ici d'un changement de para-

\footnotetext{
${ }^{1}$ Cf. M. Henry, «La critique du sujet» dans Phénoménologie de la vie, t. II : De la subjectivité, Paris, PUF, «Epiméthée », 2003, p. 9-24; ainsi que «Phénoménologie de la vie» dans Phénoménologie de la vie, t. II : De la phénoménologie, Paris, PUF, 2003, qui contient le rapport illustré avec l'évangile de Jean (p. 75 sqq.).

${ }^{2}$ Cf. pour cette discussion R. Kühn, Radikalisierte Phänomenologie, Francfort/M., Peter Lang, 2003 ; H.-D. Gondek et L. Tengelyi, Neue Phänomenologie in Frankreich, Francfort/M., Suhrkamp 2011, p. 114-151 sur M. Henry.

${ }^{3}$ Dans un autre article sur le rapport entre phénoménologie et christianisme, le concept de «congruence " est également utilisé ; cf. M. Henry, Phénoménologie de la vie, t. IV, op. cit., ici surtout p. 103 sqq.

${ }^{4}$ Cf. R. Welten, «God is Life. On Michel Henry's Arch-Christianity », dans P. Jonkers et al. (dir.), God in France. Eight Contemporary Frend Thinkers on God, Leyden, Peeters 2002, p. 119-142.
} 
digme épistémologique dans le cadre d'une discussion autour de la vérité, mais de son décalage principiel. Et ce pour autant que la discussion gnoséologique soit, la plupart du temps, encore déterminée par un héritage historico-herméneutique ou analytique : histoire et langage — tout comme raison ou discours — ne peuvent pourtant pas asserter cette « vérité » qu'obtient la phénoménologie matérielle, dans la mesure où les structures de rapports intentionnels dans l'horizon du monde restent nécessairement idéalisants et, ainsi, irréalisants — ce qu'avait déjà montré Husserl dans sa critique du psychologisme et du scientisme.

\section{2/ Ipséité et salut dans le Premier-Vivant}

Si la Vérité reste, dans sa manifestation, concrètement liée à une possibilisation vivante affective ou charnelle, alors seule la vie en tant qu'elle forme un tel fondement auto-affectif et incarné peut s'asserter soi-même comme vérité. Cela signifie (selon le point de vue de l'archi-christologie) que tout discours de la théologie ou de la religion de la philosophie à propos $d u$ Christ, que l'adoption éternelle ou transcendantale du Christ est toujours déjà co-fondée dans notre auto-affection propre avant que nous la reconnaissions comme «Verbe divin » dans les Ecritures. ${ }^{1} \mathrm{Si}$ le Christ est « celui » qui « rend vivant», alors cette vivification ontologique radicale, de même que notre entrée dans l'auto-révélation toute aussi vivante de Dieu, doit posséder une réalité qui ne s'épuise pas ni se fonde en une simple proposition, laquelle se tient dans l'horizon du monde et de son langage comme système de référence. L'eucharistie est un bon exemple pour la nécessité d'un changement de pensée méthodique : «Faites ceci en mémoire de moi.» Seule l'exécution ou l'agir confère ici à la parole eucharistique sa capacité concrète de transformation, et cela prouve à nouveau et phénoménologiquement que l'analyse cohérente de la vie et de ses modes d'exécution donne un cadre pratique à la compréhension du christianisme $^{2}$, rejoignant par là l'essence et l'effet du credo chrétien dans sa nature également sacramentale, afin de penser « l'Archi-intelligibilité » «par-delà phénoménologie et théologie $» .^{3}$

Il doit alors être possible de repérer dans le prologue de Jean une rupture ou une épochè de toute généalogie mondaine, et qui concernerait non seulement l'être-né transcendantal de l'homme mais aussi l'archi-génération du fils de Dieu. C'est la raison pour laquelle Henry débute son exposition de Jean 1, 1-18 (ce qu'il nomme

\footnotetext{
${ }^{1}$ M. Henry a repris ce problème dans Paroles $d u$ Christ, Paris, Seuil, 2002 ; cf. Ph. Capelle (dir.), Phénoménologie et christianisme chez Michel Henry. Les derniers écrits de Michel Henry en débat, Paris, Cerf, 2004, avec des réponses de M. Henry par rapport à la question de l'écriture et de la tradition dans une optique phénoménologique.

${ }^{2}$ Cf. R. Kühn, Gabe als Leib in Christentum und Phänomenologie, Wurzbourg, Echter, 2004.

${ }^{3}$ Tel est l'intitulé de la conclusion de Incarnation, p. 361-374: «Par-delà phénoménologie et théologie : l'Archi-intelligibilité johannique ».
} 
une "phénoménologie du Christ ») par l'affirmation selon laquelle la relation humaine entre père et fils ne sera jamais une allégorie pour l'auto-révélation divine. Le fils de Dieu est lui-même au commencement, alors que le fils humain sera toujours précédé par un père sans que ce rapport d'antériorité ne puisse être infirmé. Or, ce n'est pas seulement l'irréversibilité de ce rapport qui est abolie dans le prologue de Jean, mais aussi cette différence qui domine le rapport entre père humain et fils humain. Car l'extériorité à l'œuvre dans cette distance irrécupérable entre père naturel et fils signifie, finalement, l'extériorité du monde lui-même en tant qu'elle est son mode de manifestation originel en raison duquel tout étant qui apparaît en lui, que ce soit un être humain ou une chose, reste extérieur à l'autre. Le prologue de Jean opère de prime abord la rupture avec cette condition de manifestation la plus générale qui ne se laisse pas contourner dans le monde en tant que tel. Cette rupture s'accomplit en situant la naissance et la vie en deçà d'un tel monde et de sa vérité ek-statique, c'est-à-dire dans "l'intériorité de l'expérience transcendantale », ce qui implique par ailleurs une mise entre parenthèses de toutes les fonctions vivantes et organiques au profit de la vie auto-affective ou génératrice qui les rend possibles. ${ }^{1}$ Par la même occasion sont mises entre parenthèses les approches scientifiques d'une vie simplement biologique, chimique ou neuronale puisque, de même que l'épreuve vitale naïve, elle n'atteignent jamais l'auto-génération originaire impliquant toujours une auto-révélation dans l'ipséité première ou essentielle.

La raison pour laquelle le verset 1,13 du prologue de Jean n'admet pas la génération sexuelle comme une compréhension adéquate de la naissance archi-originelle ne tient pas à un quelconque défaut moral du « sang » ou de la « chair », mais relève du fait que toute " génération » humaine (à l'œuvre dans le rapport entre père et fils) présuppose de manière immanquable la vie. L'évocation de ceux qui «sont nés de Dieu », au même verset 1,13 , fait donc signe vers une archi-naissance plus ancienne que la leur, qui concerne le Logos et, par conséquent, la révélation du Verbe en Dieu lui-même comme génération de ce Verbe dans le sens de l'archi-fils. Par la réduction phénoménologique et transcendantale de la naissance ou de la généalogie mondaine, le prologue de Jean parvient à réduire de la même manière toute temporalité, de sorte que Dieu, le début et la génération de l'archi-fils comme Logos ou autorévélation divine, deviennent un. Cela implique que la vie originellement absolue en tant que « Père » n'est possible que parce qu'elle contient en soi sa propre révélation en tant qu'auto-génération, c'est-à-dire l'ipséité du premier-vivant « au commencement », et en même temps en tant que commencement, puisque tout devenir ne peut se développer qu'en lui ${ }^{2}$. Cela étant, l'ipséisation de la vie divine est non seulement aussi «vieille» qu'elle-même, mais plus encore, elle ne désigne plus une quelconque différence, transcendance ou extériorité au sein de ce que Henry nomme une « étreinte » archi-originelle. L'être-chez-Dieu est donc identique à l'être-Dieu lui-

\footnotetext{
${ }_{1}^{1}$ Cf. M. Henry, C'est moi la vérité, op. cit., p. 99 sq.

${ }^{2}$ Jean 1,1 et 3 .
} 
même et c'est pourquoi le noyau dur du christianisme est constitué par cette " tautologie » entre début/Dieu/Verbe. Et si Henry fait remarquer que tout être-chez-Dieu du Logos « est déjà arraché à la longue suite des contresens que va lui faire subir la pensée occidentale jusqu'au bei sich hégelien ${ }^{1}$, alors nous sommes capables de saisir toute la pertinence phénoménologique du prologue de Jean en tant qu'il fonde l'identité entre vie et ipséité en une immanence atemporelle (ou « intériorité réciproquement phénoménologique ») du Père et du Fils, tant pour la religion chrétienne que pour une pensée conforme à la phénoménologie : «En lui était la vie » (Jean $1,4)$.

Comme à Maître Eckhart et à Fichte, Henry accorde également au prologue de Jean une puissance intellectuelle, qui non seulement lui permet d'achever ses premières recherches sur l'auto-affection ${ }^{2}$, mais qui laisse de plus entrevoir la fragilité de toute ek-stase, de la mondanéité temporelle qui ne parvient pas à se fonder ellemême - abolissant de ce fait l'apparente autonomie intentionnelle des rapports ou processus de la conscience humaine. Si le processus d'auto-génération de la vie en tant que "génération du Verbe » n'est pas antérieur à cette dernière, alors cette auto-génération comme auto-révélation de Dieu contient en soi et comme sa condition interne propre l'ipséité du premier-vivant - et réapparait dans la génération de tout vivant au sein de ce premier-vivant dans la mesure où nous, les hommes, sommes nés pareillement par le commencement de l'incarnation éternelle du Fils. La relation du premier-vivant à la vie (au Père) n'est donc pas contingente, car la vie ne peut se développer sans son Verbe qui lui est originairement indigène. D'après Jean 1, 18 - la fin du prologue — le «Fils unique » reposerait dans le sein du Père, c'est-à-dire dans le Père, de la même manière que le Père repose en son Fils, et cette « intériorité réciproque » traversera dorénavant l'intégralité du quatrième évangile. Les discours par lesquels le Christ tente de mettre en question les rapports mondains ne concernent alors pas uniquement un ordre éthiquement ou existentiellement inachevé. Henry souligne avec raison qu'il s'y agit chaque fois de la condition ontologique de notre naissance transcendantale dans la vie par le premier-vivant, le Christ lui-même. Etant donné cette naissance invisible qui est pourtant notre origine phénoménologique essentielle, ces relations qui se créent de manière familiale ou so-

\footnotetext{
${ }^{1}$ M. Henry, C'est moi la vérité, op. cit., p. 101. Rappelons que l'interprétation «objective» et « subjective » du rapport entre Dieu et Logos ne joue un rôle qu'en tant que relation de la réflexion au réfléchi présupposé, de sorte que le «auprès de Dieu » (bei) ne concerne pour le Logos que la séparation entre forme et matière en Dieu. Cf. G. W. F. Hegel, Der Geist de Christentums, in: Frühe Schriften (Werke I), Francfort/M., Suhrkamp, ${ }^{3} 1994$, p. 373 sq. Dans les leçons plus tardives

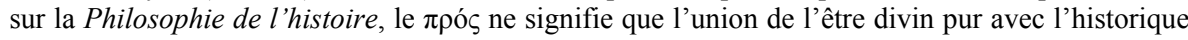
dans le Christ. (cf. Philosophie der Geschichte, Francfort/M., Suhrkamp, 1970, p. 401).

${ }^{2}$ Ce qui s'ajoute à la vie absolue de l'affectivité transcendantale de L'essence de la manifestation est la nécessité d'un premier «soi» pour la réalisation de l'absolu dans sa simplicité en tant qu'essence de la manifestation. Ainsi, les dernières œuvres de Henry (comme C'est moi la vérité ou Incarnation) ne témoignent pas d'un « tournant théologique » de sa phénoménologie.
} 
ciale autour de l'être-père mondain ne constituent plus la référence dernière à la réalité et sont donc suspendues par le Christ : seul celui qui fait la volonté divine lui est frère, sœur ou mère. ${ }^{1}$

De ce retournement radical des rapports mondains résulte, en fin de compte, la logique ferme de la religion chrétienne, exprimée par la phénoménologie matérielle en accord avec la «phénoménologie jusqu'à présent inaccomplie» de l'évangile selon Jean : la venue du Christ a pour but de permettre à l'homme la re-connaissance de son vrai père dont il a perdu la mémoire dans son intérêt unique pour le monde. ${ }^{2} \mathrm{Le}$ dépassement de cet oubli est identique au salut que Jésus annonce et réalise dans sa forme de Christ en témoignant pour le Père essentiellement invisible mais originaire, et ce dans le mode de manifestation mondain - dans son « extériorité » ou dans son « souci ». Cette constellation de connaissance d'intervenir au sein du domaine de la visibilité mondaine en faveur de la «paternité » archi-originelle de la vie absolue, cela est identique à la situation phénoménologique par excellence : ce qui semble être non-manifeste (Dieu) représente la phénoménalité authentique (la vie) cachée par la structure de la manifestation mondaine parce que celle-ci lui est impossible, dans la mesure où elle est « conscience de... », de saisir la vie divine - et ainsi doit l'oublier sans cesse. ${ }^{3}$ Le caractère inouï et phénoménologiquement unique du christianisme d'après le prologue de Jean tient en ceci que cette situation de connaissance décisive à propos du salut n'est pas assimilable par un quelconque savoir gnostique, mais qu'elle trouve justement son accomplissement en cette unité christologique entre « splendeur divine » et « incarnation du Verbe».

La « re-connaissance » de la paternité divine comme étant notre salut tel qu'il est proclamé et réalisé par le Christ ne serait donc pas un événement intellectuel ou spirituel à la manière d'un quelconque effort intellectuel, mais reposerait au sein de l'unité de la révélation divine et de l'être-fils du Christ en ce qu'elle est Verbe dans la chair. La situation dramatique qui oppose la phénoménologie du monde et la phénoménologie de la vie se déplace alors en direction d'une problématique en deçà de tout les accès théoriques à la connaissance, puisque chaque «chair » est avant tout et en raison de son incarnation originaire auto-épreuve. La « gloire » dont il est question en Jean 1, 14 et 18, est la gloire du Père en son archi-fils, et, par là même,

${ }^{1}$ Cf. Math 10, $43 s q$ et $12,48 s q$.

${ }^{2}$ Cf. M. Henry, C'est moi la vérité, op. cit., p. 103 sq.

${ }^{3}$ Que ceci représente un «tournant » réciproque de la théologie et de la philosophie confirme la position de Jean-Luc Marion dans Figures de phénoménologie. Husserl, Heidegger, Levinas, Hen$r y$, Derrida, Paris, Vrin, 2012. Et cela souligne notre effort de voir dans cette réception philosophique du prologue de Saint Jean une nouvelle forme de «phénoménologie religieuse ».

${ }^{4} \mathrm{C}$ 'est une question critique à poser à l'adresse de l'interprétation de J. Hatem, Le sauveur et les viscères de l'être. Sur le gnosticisme de Michel Henry, Paris, L'Harmattan, 2004. Cette problématique gnostique est mieux vue par A. Navigante, "Gnostische Wahrheit und christliche Offenbarung. Anmerkungen zu Michel Henrys Hyletik des Lebens» dans K. Appel, J.B. Metz u. J.-H. Tück (dir.), Dem Leiden ein Gedächtnis geben. Thesen zu einer anamnetischen Christologie, Göttingen, V\& R unipress, Vienne, 2012, p. 379-401. 
l'autorévélation de la Vie divine en son commencement absolu antérieur au monde et au temps. Si l'incarnation du Christ se doit donc de réaliser la révélation divine "pour les hommes », elle ne pourra le faire qu'à travers l'être-charnel propre aux hommes en tant que cette naissance de notre venue-en-vie est pareillement donnée avant toute temporalité. Jésus entre dans la visibilité du monde en tant qu'être humain possédant un corps visible qui ne se distingue pas des autres corps humains puisqu'il participe pareillement au phénomène du monde. Ainsi, la certitude à propos du «salut» en Christ nous est inaccessible aussi longtemps que nous passons par la perception mondaine ou visible, et ce en raison du manque d'un critère absolu au sein du monde et de la conscience pour cette certitude de la vie absolue en et par le Christ. Le déplacement qui s'est opéré au sein de la situation phénoménologique initial en rapport à la question de la re-connaissance de notre salut concerne pour cette raison non seulement le déplacement de l'horizon du monde en tant qu'espace de manifestation externe vers une épreuve auto-affective de la chair comme immanence radicale ou originelle au sein de l'être-fils du Christ, mais aussi le déplacement qui avec lui va de pair : celui de la problématique de l'attestation, en tant que le Christ ne l'attend plus des hommes mais uniquement de son Père. C'est pourquoi la question du témoignage telle qu'elle figure dans l'évangile de Jean et a été analysée par Henry ${ }^{1}$ devient la question principale de l' " archi-intelligibilité johannique » elle-même.

En accomplissant ce déplacement, la méthode ainsi que l'objet d'une "philosophie de la religion » sous forme de phénoménologie de la vie originellement «incarnationnelle » se transforme : la gloire du fils « dans le sein du Père » n'est donnée invisiblement qu'à la lumière du monde visible, de sorte que même une telle " invisibilité » permet de reconnaitre la vie du Fils dans le Père et, vice-versa, du Père dans le Fils. Aussi longtemps que cette invisibilité est autant plénitude de la grâce que vérité, elle contiendra en elle la réalité de Dieu lui-même et son autoenveloppement dans le Fils, c'est-à-dire son auto-révélation dans le Verbe, antérieure au premier témoignage de saint Jean Baptiste. ${ }^{2}$ La problématique du témoignage en rapport au Christ est donc thématisée de manière principielle en ce que la manifestation visible (la « descente de la colombe ») est annulée, car la visibilité de la colombe ne remplace pas le fait que Dieu lui-même atteste la vérité de cet événement, c'est-à-dire la lie à son auto-révélation : « Celui qui m'a envoyé baptiser dans l'eau, c'est lui qui m'a dit : “Celui sur lequel tu verras l'Esprit descendre [...], c'est lui" ». ${ }^{3}$ L'attestation de la révélation du premier-vivant peut donc être prise en charge uniquement par la vie et son auto-révélation en tant que telle, c'est-à-dire, en ce cas-ci, par la «diction divine» comme révélation de l'adoption du Christ. Le témoignage face aux Pharisiens part de la même structure d'invisibilité : « Au milieu

${ }^{1}$ Cf. C'est moi la vérité, op. cit., p. 113 sqq.

${ }^{2}$ Jean $1,15$.

${ }^{3}$ Jean 1,33 sqq. 
de vous se tient celui que nous ne connaissez pas. $»^{1}$ De même, la manifestation visible du Christ ne suffit pas, dans le cas du miracle de la guérison de l'aveugle-né et de l'entretien avec Philippe, à le faire reconnaitre en tant que messie et fils de Dieu. $^{2}$ Cette situation ne se répète pas seulement lors des rencontres avec Jésus, mais contient, selon le prologue de Jean, la situation de base de la foi en tant que renversement de la problématique phénoménologique du témoignage: le Christ témoigne de lui-même aussi longtemps que son immanence témoigne pour luimême en son Père.

\section{3/ Foi et vie au sein de l'archi-christologie}

La foi, qui embrasse cette intériorité de l'archi-fils en Dieu comme origine de la Vie et qui re-connait donc ce rapport en l'homme transcendantal lui-même, cette foi n'est donc ni un moindre «savoir » que le savoir mondain, ni moins parfait ou moins accompli que celui-ci, malgré ce qu'une vue intentionnelle pourrait nous faire croire. Bien plutôt, la foi concerne par principe un autre ordre de manifestation puisque la loi mondaine de la visibilité ne lui est pas homogène et ne peut donc pas être sa mesure. Cette «substitution» de l'ordre du monde à un « ordre plus essentiel », comme l'appelle Henry ${ }^{3}$, non seulement ôte à la foi sa structure d'incomplétude apparente, mais met également entre parenthèses l'attente habituelle liée à toute autre foi comprise comme «présomption» (Vermeinen), afin de mettre en avant l'auto-donation absolue de la révélation en tant qu'elle est donnée avec la révélation du Verbe de la vie comme vie de l'archi-fils dans son Père. La « théologie du présent » infaillible au sein de l'évangile de Jean n'est donc pas simplement un attribut littéraire de celui-ci, mais bien plutôt la révélation divine en tant que présence. Dans cette invisibilité face au monde se manifeste la destinée phénoménologique nécessaire de la foi pour autant qu'elle est reconnue en son identité immanente avec le processus de vie absolue: dans la foi en tant que telle s'atteste l'autocroyance de la vie en elle-même puisqu'un tel auto-engagement de la vie par rapport à elle-même laisse entrevoir, de par sa manière d'être indestructible, l'intériorité réciproque ou mieux le dévouement vital du Père et du Fils. Dans le cas où l'épochè de la visibilité mondaine signifie en même temps la mise entre parenthèses de toute thématisation ou protention intentionnelle en tant qu' « attente », la foi est égale à ce processus de vie lui-même. La détermination henryenne d'une phénoménologie de la «philosophie de la religion chrétienne » se laisse maintenant comparer sans problèmes à la position fichtéenne ou biranienne. La foi se situe, de par sa structure phénoménologique radicale, en deçà de toute herméneutique. Elle est l'autorévélation de la vie elle-même, telle l'immédiateté du savoir qu'a Jésus de soi d'être

\footnotetext{
${ }^{1}$ Jean $1,16$.

${ }^{2}$ Jean 9,35 sqq. et $14,16 s q q$.

${ }^{3}$ Cf. C'est moi la vérité, op. cit., p. 108 sq.
} 
le fils de Dieu, c'est-à-dire d'être le « docteur interne » du « soi interne » ou purement spirituel qui s'éprouve dans la descendance absolue d'une affection passible de la vie. ${ }^{1}$

Il ne s'agit pourtant pas pour Henry d'instaurer une autre « christologie spéculative » comme celles qui furent tentées, bien avant l'idéalisme allemand, dans les recherches théologiques inspirées des néoplatoniciens ou encore d'Aristote. Il est bien plutôt question de cette archi-christologie, qui précède nécessairement tout effort intellectuel, et qui se présente dans le texte de Jean sous forme de cette identité entre authenticité et vérité : ce que dit le Christ de lui-même correspond non seulement à la vérité historique mais implique également la vérité pré-temporelle et acosmique de ce dont Jésus témoigne ainsi par lui-même. Car même si Jésus a parlé subjectivement et objectivement de lui-même en tant que «Fils de Dieu », ce témoignage ne serait toutefois pas un critère suffisant pour prouver qu'il est «premiervivant » - son amour pour Dieu ainsi que son propre enthousiasme auraient pu être à l'origine de telles déclarations sans nécessairement réaliser son être immanent, celui d'être effectivement «Verbe divin» dans le sens de l'auto-révélation divine archi-originelle. ${ }^{2}$ La problématique du témoignage dans l'évangile de Jean mis en évidence par Henry doit donc correspondre à une telle archi-christologie dans le cadre de laquelle les écrits johanniques ne représentent pas un simple récit de communauté, mais désignent l'évidence avec laquelle Jésus se comprend comme Verbe divin. Voilà pourquoi ce qui suit après le prologue de Jean doit être compris comme l'expression de l'unité entre authenticité et vérité intérieure de l'auto-témoignage du Christ: «Les paroles que je vous dis, je ne les dis pas de moi-même ! Au contraire, c'est le Père qui, demeurant en moi, accomplit ses propres œuvres. Croyez-moi, je suis dans le Père et le Père est en moi. $»^{3}$

Ces déclarations en première personne faites par Jésus, dont les paroles du prologue font également partie, ne peuvent être comprises que si l'on reconnaît en même temps le «tournant» opéré au sein des conditions de vérité phénoménologiques exprimant l'accès au contenu de la foi chrétienne. Et ce qui exige d'être retourné - nous l'avons mentionné en étudiant la problématique du témoignage -

\footnotetext{
${ }^{1}$ Pour Fichte cf. son Introduction à la vie bienheureuse, $6^{\mathrm{e}}$ leçon avec l'appendice ; pour Maine de Biran, cf. ses commentaires de l'Évangile de Saint Jean dans Euvres, t. X-1 (éd. Azouvi), Paris, Vrin, 1987, p. 149-163, ainsi que « Nouvelles notes sur l'Évangile de Saint Jean », dans Euvres, t. XIV (éd. Tisserand), Paris, PUF, 1949, p. 429-435.

${ }^{2}$ Cf. M. Henry, «Archi-christologie », dans Phénoménologie de la vie, t. IV, op. cit., p. 113-129. Henry se réfère ici à C. Tresmontant, Le Christ hébreu, Paris, Albin Michel, 1992, qui place Jean dans le cercle théologique autour du prêtre de Jérusalem ce qui, en tant que disciple de Jésus, lui donnait immédiatement accès aux controverses juridiques et théologiques des autorités religieuses. Les ipsissima verba de Jésus à propos de son adoption divine relèvent donc du témoignage de Jésus lui-même dont le caractère dramatique et l'authenticité consistent dans leur issu : la mort du Christ. Seul ce dernier rapport nous préoccupe ici, c'est-à-dire la vérité absolue ou divine du Christ en tant que tel.

${ }^{3}$ Jean $14,10 s q$.
} 
n'est autre que le rapport entre réalité mondaine et réalité de la vie : cette dernière abolit la position prééminente de la philosophie traditionnelle grecque (et, plus tard, rationnelle classique) selon laquelle la vérité n'est possible qu'à travers la phénoménalité de la visibilité, c'est-à-dire la lumière de la perception et de l'intellect. Ce logos mondain n'est pourtant pas détruit, il reste valable uniquement pour le domaine du savoir objectif et « horizontal» qui n'a pas de validité pour la vérité interne du Christ (y compris sous la forme de l'histoire ou de l'herméneutique). Au moyen de cette annulation des catégories mondaines qui sont, apparemment, les seules catégories déterminantes, ainsi que des rapports humains qui y sont impliqués, le Christ ouvre une autre voie d'accès à l'« épreuve » de la réalité de la vie qu'il est lui-même. Condition et épreuve de vérité se réunissent ainsi en Christ : «C'est moi la vérité. » / « Je ne suis pas du monde. »/ «Au commencement était le Verbe. $»^{1}$ Le Christ, et après lui le christianisme, effectuent donc un retournement radical des concepts phénoménologiques et de l'expérience selon lesquels se forme traditionnellement la pensée.

Ceci se laisse comprendre immédiatement au moyen du rapport lumière/obscurité, rapport mis en avant par le prologue de Jean en tant que « connaissance croyante » du Christ, comme étant déterminant pour le reste de l'évangile. ${ }^{2}$ L'équivalence entre perception, monde et vérité n'habite pas seulement toute pensée quotidienne ou scientifique, mais aussi, sous forme de «transcendance », le dire religieux habituel - ce qui, par principe, bannit la vie divine dans une extériorité ou distance à laquelle s'ajoute occasionnellement (comme chez saint Augustin) ou «mystiquement » (comme chez Maître Eckhart) l' " intériorité », c'est-à-dire cette immanence divine dans l' «âme». Le prologue de Jean «néantise » la simple vérité mondaine comprise comme lumière qui, au moment de l'arrivée du Christ en un tel monde lumineux, se transforme en l'exact opposé, l'obscurité. En effet, la « vraie lumière » qui « en venant dans le monde, illumine tout homme $",{ }^{3}$ ne guiderait que vers une seule lumière illuminant toute chose si cette «venue» n'engendrait pas par ellemême sa condition de connaissance constitutive propre. Car si le Verbe divin archioriginaire vient dans le monde, cela suppose qu'il n'en fait pas partie, sans quoi il devrait s'épanouir dans la lumière d'un tel monde, c'est-à-dire être depuis toujours identique avec lui. L'archi-intelligibilité johannique brise justement cette présupposition qui semble aller de soi et transforme la lumière mondaine en obscurité. Ainsi, le retournement radical susmentionné est réalisé et il doit être compris comme la fondation d'une phénoménologie nouvelle pour la foi chrétienne ainsi que pour une philosophie de la religion, c'est-à-dire comme une archi-christologie purement phénoménologique avec ses constellations particulières de témoignages et de vérités propres. La lumière du monde n'est pas en soi obscurité puisqu'elle continue à illu-

\footnotetext{
${ }^{1}$ Jean 14,$6 ; 17,14 ; 1,4$.

${ }^{2}$ Cf. C'est moi la vérité, op. cit., p. 110 sq.

${ }^{3}$ Jean 1,9 .
} 
miner tout étant afin de le faire connaître ; mais cette puissance d'horizon devient impuissante face à l'auto-révélation de la Vie en tant qu'archi-fils divin : "En lui était la vie et la vie était la lumière des hommes. ${ }^{1} \mathrm{Si}$ la lumière du monde «ne comprend pas » cette lumière de la vie tout en y luisant, alors cette métamorphose de lumière en obscurité au moment où apparaît la vraie lumière manifeste un nouveau mode de phénoménalisation principiel : «Moi, la lumière, je suis venu dans le monde afin que quiconque croit en moi ne demeure pas dans les ténèbres. $»^{2}$

Seule la distinction critique opérée par la phénoménologie matérielle entre deux modes de révélation - phénoménalité du monde et phénoménalité de la vie ${ }^{3}$ — est capable d'expliquer ce fait que Jésus apparaît comme un homme de ce monde tout en se réclamant d'une essence éternelle et divine en raison de la réalité de sa révélation comme logos ou « Verbe divin ». Le dessaisissement de sa condition divine ${ }^{4}$ est d'une telle radicalité qu'il est $v u$ incontestablement dans ce monde en tant que Jésus de Nazareth, mais que la plupart des gens ne croient point en lui. ${ }^{5} \mathrm{Si}$ cet autodessaisissement divin entrepris dans le but d'une soumission aux conditions du monde phénoménal est si radicale qu'elle entraine l'exclusion de la foi du registre de la vue - et de la vue hors de celui de la foi - alors se révèle soudainement tout le drame d'une «phénoménologie du Christ» comme « archi-christologie ». D'un point de vue mondain, d'un point de vue d'une religion de la transcendance absolue, la revendication d'exister comme le «Verbe divin» sous la condition d'un autodessaisissement doit mener à l'accusation de blasphème, ce qui est presque inévitable face à un tel incognito nécessaire. ${ }^{6}$ Il n'existe pourtant pas qu'un seul accès à la vérité du Christ à travers la vie invisible et son auto-affection comme auto-étreinte, car même si ce contexte est révélé, il faut retenir, en un sens johannique, qu'il y a une unité originelle entre la vie divine et le premier-vivant. Le Christ n'est pas «dans » la vie à la manière dont les choses sont «dans» le monde. Par l'archigénération du Fils s'accomplit en même temps la venue-en-soi de la vie en tant que réalisation de soi-même (Père) en une ipséité première (Logos). Pour l'homme et sa foi, il n'existe de ce fait plus d'autre moyen d'accéder à la vie ou au salut que de s'étreindre passiblement «dans le mouvement de cette auto-étreinte de la Vie»,

\footnotetext{
${ }^{1}$ Jean 1,4 .

2 Jean 12,46

${ }^{3}$ Pour une discussion du contexte phénoménologique cf. M. Henry, Phénoménologie matérielle, Paris, PUF, 1990, p. 61-136 : «La méthode phénoménologique «; Incarnation, partie II : «Phénoménologie de la chair », comme application à la pensée grecque (gnostique) et chrétienne.

${ }_{5}^{4}$ Cf. Philippe 2, $6 s q$.

${ }^{5} \mathrm{Cf}$. Jean, 6,36 .

${ }^{6}$ Ce motif du incognito est repris de Kierkegaard; cf. déjà, M. Henry, La barbarie, Paris, Grasset, 1987, p. 218 sq, ainsi que N. Hatem, « Le Secret partagé : Kierkegaard - Michel Henry », dans J-F. Lavigne (dir.), Michel Henry. Pensée de la vie et culture contemporaine, Paris, Beauchesne, 2006, p. $195-210$.
} 
selon l'expression de Henry, c'est-à-dire par l'ipséité essentielle du premier-vivant en tant que Verbe divin. ${ }^{1}$

Ceux qui croient en Christ, selon la pensée johannique, ne sont certes «pas de ce monde », mais l'accès à la vie dans l'auto-enlacement originel de Père et Fils devient de plus pour eux un tout nouveau mode de phénoménalisation, à savoir la pure immanence d'une telle « intériorité » en deçà de toute distance ou transcendance. Ils ne « croient » donc pas simplement en évitant toute sorte de visée ou d'attente intentionnelle, mais ils vivent dans la réalité d'une telle croyance qui est l'épreuve essentielle intérieure du Fils par excellence, et portent ainsi en eux-mêmes la condition de la vie divine comme leur propre expérience réelle grâce à l'adoption du Christ. Les écrits johanniques parlent constamment de ce mouvement sans fin en Dieu par lequel s'étreignent le Père et le Fils de manière auto-générante dans «toutes leurs œuvres », et auxquelles appartiennent également ceux qui «sont nés de Dieu ${ }^{2}{ }^{2}$ Dans le registre d'une telle archi-christologie, il faut alors que la parole du Christ revienne à son propre «Ego » engendré comme ipséité essentielle, que la vie s'éprouve elle-même et se révèle incessamment. Sa vie s'auto-éprouve comme l'auto-épreuve de la vie divine, c'est-à-dire comme le mode incessant d'autoaccomplissement de cette vie dont la « gloire » est la révélation du Père — et, par là même, « cette gloire que, Fils unique plein de grâce et de vérité, il tient du Père ». ${ }^{3}$ De sorte que le dire, l'action et la volonté du Christ ne peuvent être, en raison de cette appartenance du Fils au Père, de cette ipséisation donc, que la volonté ou l'action du Père lui-même : "Tout ce que possède mon Père est à moi. 》

$\mathrm{L}^{\prime}$ « intériorité réciproque » entre Père et Fils ne consiste donc pas simplement en cet auto-génération par auto-étreinte; leur vouloir est essentiellement un, de sorte que l'ipséité du Fils ne peut être rien d'autre que l'auto-accomplissement du mouvement de la vie divine en tant que telle. Un exemple en est la guérison lors du sabbath, l'expression immédiate d'un être divin menant à une accusation de la part des autorités religieuses mais impliquant, pour le bienfaiteur, la confirmation de son unité volontaire avec le Père - jusqu'à l'heure de la dispersion : «Je ne suis pas seul, le Père est avec moi ». ${ }^{4}$ L'identité de la volonté avec le Père, qui existera également pour le croyant, est, avant toute pertinence éthique, une réalité identitaire ontologique, celle-là même qui se trouve exprimé par Jean et les synoptiques sous le mode de l'apodicticité. ${ }^{5}$ S'il n'existe pas deux volontés en Christ, ce que Fichte souligne déjà à propos de l' " agir pur » (reines Tun), alors tout se réalise en Christ en tant qu'essence de l'unité divine elle-même - ce qui entrainera un retournement phénoménologique du concept de volonté puisque celle-ci ne s'orientera plus selon

\footnotetext{
${ }^{1}$ Cf. C'est moi la vérité, op. cit., p. 113.

${ }^{2}$ Jean 1,13 .

${ }^{3}$ Jean 1,14 .

${ }^{4}$ Jean 16,32 ; cf. 10, 38 .

${ }^{5}$ Cf. Luc 10, 22 : « tout m'a été remis par mon Père ».
} 
une loi extérieure. En tant qu' « envoyé divin » en identité avec Dieu, le Christ comprend les écrits (Thora) sans avoir été instruit spécifiquement ${ }^{1}$; et ce qu'il dit doit nécessairement être l' " enseignement divin » lui-même, c'est-à-dire son « Verbe ». ${ }^{2}$

Jean reconnaît en la «personne» du Christ, en cette ipséité essentielle, l'événement de vie par excellence et fait de cette vérité de base le noyau dur de la foi chrétienne. Le Christ, en tant qu' « envoyé divin », puise la condition de cet envoi en Dieu lui-même, dont il est l'œuvre et l'enseignement, dans la mesure où celui qui l'a envoyé dit la vérité qu'il garantit par l'unité avec le Christ en tant qu'il est son Verbe. ${ }^{3}$ Tout essai de compréhension d'une telle vérité de la vie en son autorévélation réciproque interne par le logos du monde et de ses étants doit, pour des raisons purement phénoménologiques, échouer face à cette vérité centrale de la vie et qui consiste à être partout et toujours elle-même et à se révéler ainsi seul par et en elle-même - dans l'ipséité du premier-vivant de la vie dont la parole est identité avec le Verbe de la vie qui s'accomplit de manière auto-révélatrice en cette parole. La réponse à la question - celle de savoir comment Jésus de Nazareth « montre» la vérité de son être-Christ - , ne peut de ce fait que consister en un renvoi au processus immanent d'auto-révélation de la vie, identique, en tant que phénoménalité de la vie, à la phénoménalisation intradivine du processus d'auto-génération de la vie. La manifestation du Christ dans le monde, son épiphanie en elle, ne correspond donc pas à la monstration ou à l'être-vu mondain, mais signifie l'unité de la " gloire » de Dieu et du Christ qui, étant un événement de vie transcendantal, "glorifie» le Fils jusqu'à la passion de Jésus. ${ }^{4}$ Distincte de l'« honneur du monde» qui ne cherche qu'elle-même et ne s'occupe que d'elle-même, l'archi-fils ne connaît que l'honneur de celui qui l'a envoyé, l'origine de la vie auquel seul revient tout honneur. ${ }^{5}$

La foi qui honore Dieu en tant qu'origine de la vie « avant tout monde » est donc identique, en raison de l'intériorité phénoménologique du Père et du Fils, à la foi en Christ puisque c'est uniquement en lui que le Père peut être «vu» alors qu'il échappe à toute vue. ${ }^{6}$ Or, parce que le Christ en tant que premier-vivant ne peut être vu ou connu dans le monde en tant que tel, parce que tout signe mondain doit être mis entre parenthèses en raison de sa vérité7, la vérité d'une telle vie acosmique et autarcique ne devient accessible que par la co-appartenance de son intériorité phénoménologique et de son processus de révélation : «Nul n’a vu le Père, si ce n'est celui qui vient de Dieu. $\rangle^{8}$ L'aporie de la perception de Dieu ne se résout donc pas par la perception que nous avons de Jésus dans le monde, puisqu'une telle percep-

\footnotetext{
${ }^{1}$ Jean 7,15 .

${ }^{2}$ Jean 8,$28 ; 12,49 ; 14,10$.

${ }^{3}$ Cf. Jean 8, 25.

${ }^{4}$ Jean 13,31 ; cf. 17, 4 sq.

${ }^{5}$ Jean 5,41 sqq. ; 7, 17 sq.

${ }^{6}$ Jean 12,45 .

${ }^{7}$ Jean 6,30 .

${ }^{8}$ Jean 6, 46.
} 
tion ne laisse point entrevoir son appartenance interne au Père « au commencement ». Pourtant, lorsque la foi est comprise comme retournement phénoménologique de la perception mondaine, l'essence interne de Dieu et de son Verbe vivant et incarné devient accessible, en raison du fait que l'homme ne se comprend plus, dans son être transcendantal, comme un étant de ce monde. Le christianisme bouleverse de manière radicale l'essence de et l'accès véridique à la manifestation élémentaire en ce qu'il attribue à l'homme une naissance transcendantale dans l'ipséité du Fils de Dieu lui-même, afin qu'il puisse réaliser le salut par l'appartenance principielle à la vie interne divine et à son auto-révélation. Ainsi, le prologue de Jean, alliant brièveté concise et immense expression de foi phénoménologique, contient la possibilité d'une autre compréhension de Dieu, du monde et de la vie, dont la conséquence n'a jusqu'à présent pas été saisie dans toute son ampleur par la pensée occidentale. ${ }^{1}$ En effet, il permet de comprendre la vérité de l'ipséisation christologique comme vérité radicale et matérielle de tout Soi humain, renvoyant, au final, au contenu autoaffectif du processus de vie intradivin, ce que nous devons maintenant encore mettre au clair.

\section{4/ Auto-affection en tant qu'archi-intelligibilité de l'ego charnel}

En renvoyant à l'autonomie pré-historique du processus de vie en Dieu, l'archiintelligibilité johannique comme archi-christologie désigne la venue-en-soi de la vie archi-originelle par son auto-fondement grâce à son pouvoir d'auto-génération, accomplie dans l'ipséité du Christ. Si le Christ n'est pas, comme le souligne Henry², le «résultat» de ce processus, mais sa concrétion phénoménologique, alors cette auto-génération de la vie est, dans tous les cas, une activité phénoménologique, à savoir l'auto-phénoménalisation comme «épreuve » de l'auto-révélation où le dire lui-même et ce qui est dit sont une seule et même chose et forment ainsi l'autoétreinte du Père et du Fils. Ils forment chacun pour l'autre cette « subjectivité » dans laquelle s'affecte ou s' « éprouve » l'autre à chaque fois, afin d'être le « Soi » de cette auto-affection comme auto-épreuve. Le Soi n'est alors rien d'autre que la venue-en-soi de la vie sur le mode d'une ipséisation respectif, et de telle sorte que le Soi ne peut être que dans la vie et que la vie n'apparaît que comme « Soi » — autrement dit, qu'en tant qu'individuation radicale qui exclut toute vie générale ou étrangère, tant comme réalité que comme concept. L'archi-intelligibilité johannique de l'auto-génération intradivine, en tant que vérité christologique, témoigne donc d'un concept de vérité qui dépasse toute vérité " formelle », « impersonnelle» ou

\footnotetext{
${ }^{1}$ Cf. M. Enders et R. Kühn, «Im Anfang war der Logos... ». Studien zur Rezeptionsgeschichte des Johannesprologs von der Antike bis zur Gegenwart, Fribourg-en-Brisgau, éds. Herder, 2011.

${ }^{2}$ Cf. M. Henry, « Archi-christologie », art., cit., p. 120 sqq. ; F. Gaiffi, « La dimension trinitaire dans la philosophie du christianisme de Michel Henry ", dans J.-F. Lavigne (dir.), Michel Henry. Pensée de la vie et culture contemporaine, op. cit., p. 149-166.
} 
« scientifique », se plaçant au-dessus, ou même dans l'indépendance à l'égard des hommes, puisque cette vérité christologique est la vérité d'un seul «Moi»l'ipséité du Christ. Parce que le Christ témoigne, dans son unicité et originalité, de sa vérité, il est essentiellement la "voie» vers cette vérité, non pas en un sens méthodique ou ek-statique, mais comme cette immanence de chaque Soi né également comme un «Moi » dans cette vie du Christ ipséisée. ${ }^{1}$ Car « être né » dans la vie est identique à l'arrivée de la vie en elle-même qui, par une telle venue-en-soi, est immédiatement son auto-révélation comme auto-affection dans cet événement. «Voyant» le Christ et à travers lui le Père, cette «vue » ne se produit que comme individuation constante en lui, comme ipséisation s'auto-éprouvant de la vie «au commencement » et en tant que commencement avant et hors de toute temporalité.

Cette frontière conceptuelle de l'individuation humaine au sein de l'individuation christologique comme archi-affection est la facticité transcendantale de notre vie dans l'archi-intelligibilité johannique, un événement ayant lieu avant tout témoignage et toute vérité mondaine, comme nous l'avons montré plus haut à propos du Christ. Notre individuation en tant que réalité purement affective ou passible désigne alors la preuve vivante de la vérité de la réalité purement phénoménologique du Christ qui est archi-témoignage du Père lui-même : en étant affectés de manière unique par la vie, en y étant nés ou individués, nous appartenons à l'ipséité divine comme ipséisation, nous entendons la parole divine résonner dans la réalisation incessante de la naissance transcendantale de notre «adoption dans le Fils », pour citer à nouveau Paul. ${ }^{2}$ Si la génération du Fils par le Père est le témoignage du Père pour le Fils dans le Fils qui reçoit le Père comme son essence propre, alors le Fils est la connaissance divine dans l'auto-épreuve de sa propre " personne », comme ipséité $\mathrm{du}$ « Moi» de la vérité s'adressant si sublimement à nous dans l'évangile de Jean. Ce ne sont pas seulement témoignage et vérité qui, en un sens johannique, se rattachent l'un à l'autre, mais également vérité et «être-ego» comme ipséité de la « connaissance», «éprouvée» de manière vivante ou affective. Ces conditions de vérité intradivines doivent alors se retrouver dans le registre humain de la phénoménalisation de notre vie, s'il doit être également possible pour nous de connaître et de vivre l'auto-témoignage que le Christ fait de la vérité divine. Le christianisme, dans l'optique de l'archi-intelligibilité johannique comme archi-christologie, ouvre, par l'épochè de la vérité mondaine, la possibilité principielle pour l'homme de dire « Je » et de s'éprouver transcendentalement en tant que tel, dans l'auto-affection de la vie. Cette «épreuve » n'est pas une « expérience de soi» dans un sens purement psychologique ou existentiel. Il s'agit bien plutôt d'une épreuve de l'«adoption en

\footnotetext{
${ }^{1}$ Pour une discussion plus explicite, cf. R. Kühn, Geburt in Gott. Religion, Metaphysik, Mystik und Phänomenologie, Fribourg/Munich, éds. Alber, 2003, p. 16-78 : «Besoin et naissance transcendantale ».

${ }^{2}$ Rom, 8, $28 s q$.
} 
Dieu », dans l'expérience de soi-même en tant que telle ${ }^{1}$, et dans la lignée du renouvellement de l'anthropologie ou de la métaphysique sur la base d'une psychologie transcendantale première proposé par Maine de Biran.

Tout « Ego » individuel est, en raison de cette démonstration purement phénoménologique en affinité avec la pensée johannique, un Ego né de Dieu puisqu'un quelconque Ego n'existe que grâce à ce procédé inimaginable par lequel le premiervivant, le Christ du commencement, est né dans le sein de son Père pour s'y incarner, c'est-à-dire pour être la matérialité auto-affective de la phénoménalisation intradivine. Ma naissance n'étant pensable qu'en Dieu, devenir un Ego transcendantal dans son ipséité, la possibilisation concrète ou pratique d'un tel Soi doit être donnée comme impliquée par l'ipséisation divine dans le Christ ou le logos. En devenant des «Fils dans le Fils» par notre naissance pré-temporelle, nous ré-effectuons l'archi-naissance du premier-né en Dieu, cette ré-effectuation ne signifiant cependant pas une suite monotone d'un Même ontique, mais l'individuation radicale de tous les êtres humains possibles tels qu'ils sont nés dans l'archi-fils grâce à l'accès qui lui est ainsi ouvert vers la vie divine absolue. Ainsi, chaque Soi est exclusivement un rapport à soi-même rendu possible par la vie - ce qui, notons-le, avait déjà été entrevu par Kierkegaard ${ }^{2}$ - afin de constituer par un tel rapport pur à soi, également comme réalité de l'archi-fils, le rapport à soi de la vie dans le sens de tout «Moi » affecté. L'individuation comme Soi affectif se fait, autrement dit, de manière purement pathétique ou passible, et l'incarnation d'une telle archi-affection ou d'un tel pathos n'a d'autre "chair» que celle de l' «incarnation» éternelle du Christ : dans ma naissance transcendantale comme individuation impressionelle ou charnelle, je suis affecté par la chair du Christ comme auto-affection matérielle de la vie divine unique.

C'est ainsi que le prologue de Jean peut être doublement compris comme une archi-christologie. D'une part il n'est pas simplement un discours «à propos»du Christ, au sens objectif propre à toute théologie christologique. D'autre part, ce prologue enferme en soi l'immanence de la réalité du Christ comme condition de toute naissance en Dieu, ce qui transforme le commencement absolu qu'il énonce en cet « avant » éternel et à chaque fois nouveau du rapport à nous-mêmes. L'affinité et la congruence entre une phénoménologie radicale et l'intelligibilité johannique implique, autrement dit, une épochè radicale de toute vérité mondaine en tant que contre-réduction, puisqu'elle signifie l'auto-donation du Christ dans l'auto-donation strictement immanente de l' «homme ». Si un tel rapport à soi affectif n'est plus éprouvé dans l'extériorité du monde, alors ce rapport à soi dans le pathos respectif

\footnotetext{
${ }^{1}$ Cf. la présentation qu'en donne Michel Henry dans C'est moi la vérité, op. cit., p. 146 sqq., et p. 168 sqq., et ceci aussi dans le sens d'un oubli du «moi » originaire par l'illusion transcendantale $\mathrm{du} \ll \mathrm{Je} »$ intentionnel.

${ }^{2}$ Cf. de nouveau N. Hatem, «Le Secret partagé : Kierkegaard - Michel Henry », art. cit., p. 198 sqq.
} 
de la joie et de la tristesse ne se fonde pas dans le Soi de l'individu, mais dans le Christ et dans sa chair comme archi-ipséisation. En ce sens, le premier verset du prologue de Jean : « au commencement était le Verbe », compte pareillement pour Dieu et pour l'homme, car ce «commencement» advient, en tant qu'il est vie et auto-donation interne, comme la condition permanente de chaque Soi afin que, dans un tel «avant» transcendantal, il vienne en soi-même, c'est-à-dire éprouve son individuation immanente comme une pure auto-affection de la vie.

Contrairement à tout christo-logie philosophique, exégétique ou théologique, foncièrement inscrite dans le logos discursif, une archi-christologie comme celle du prologue de Jean, celle-là même qui se prolonge dans les discours en première personne du quatrième évangile, nous permet de voir en toute clarté combien notre rapport au Christ ne peut être fondé ni par la pensée, ni par le monde ou l'histoire, en tant qu'ils appartiennent tous à la même ek-stasis de la temporalité ou de la différence. ${ }^{1}$ Henry a montré que croire n'est pas identique à voir, raison pour laquelle c'est toujours le Christ lui-même qui donne accès à lui-même en faisant venir un Soi vers lui-même, dans l'archi-commencement, et ceci en le faisant, comme première ipséité, venir à soi-même comme sa propre ipséité. L'archi-christologie est donc l'auto-donation dans ce double sens phénoménologiquement inséparable : autodonation de tout Soi à soi-même dans l'autodonation intradivine du Christ comme Verbe divin. Être vivant ou être en vie dans une individuation ineffaçable n'est possible que dans une archi-christologie immanente dont les principes immuables ont été dessinés, de toute éternité, par le prologue de Jean.

Dès lors que la foi cesse d'être «attente» pour devenir présence de l'autodonation du Christ dans notre «chair» qui s'affecte en sa chair, nous ne pouvons plus nous « élever » à partir de notre « adoption » vers Lui puisque l'auto-devenir du Moi pur comme rapport à soi n'admet pas de transcendance ou de distance au sens d'un « dépassement » intentionnel. Avant d'être en nous, Il est en nous et seul cette œuvre divine immémoriale dans la nuit de notre chair affective, dans la passibilité de son auto-impression, fait une vie «en plénitude»: «Je suis la porte; si quelqu'un entre par moi [...], il ira et viendra et trouvera de quoi se nourrir. $»^{2}$ Le logos de la vie n'a pas lieu comme discours sur la vie, mais uniquement en affectant au sein de cette vie elle-même, dans son immanence entendue comme naissance

\footnotetext{
${ }^{1}$ Ainsi, il faut corriger l'interprétation de Xavier Tilliette selon laquelle il s'agirait chez Henry d'un «Christ d'une appartenance purement philosophique» tel qu'il a été médiatisé par l'«absolu pathétique » d'un Maitre Eckhart et de Novalis. Cette interprétation est encore valable pour L'essence de la manifestation de 1963, mais plus pour l'œuvre tardive, que Tilliette ne connaissait pas encore ; cf. Le Christ de la philosophie. Prolégomènes à une christologie philosophique, Paris, Cerf, 1990, p. 262. Voir aussi la contribution finale de Frédéric Seyler sur le Christ chez M. Henry dans le recueil de J.-P. Resweber (dir), Le Jésus des philosophes, Metz, Les Cahiers du Portique, 2008.

${ }^{2}$ Jean 10, $9 s q$. Pour l'interprétation de cette parabole du berger et ses brebis au sens de la naissance du Soi dans l'ipséité du Christ, cf. C'est moi la vérité, op. cit., p. 145 sqq.
} 
incessante ou comme auto-donation dans l'auto-génération absolue de la vie, c'està-dire de la chair du Christ dans le sein de son Père. Quand Jésus, dans l'évangile de Jean, parle, sans médiation mondaine, de son essence propre de Fils de Dieu afin de la partager avec nous dans les paroles de la vie, c'est-à-dire dans l'auto-donation du Soi chaque fois absolument commençante, il joint le geste à sa parole afin de sceller cette auto-donation pure comme donation de sa propre vie - par le «pain de la vie », l'eucharistie ${ }^{1}$, comme nous l'avons déjà mentionné. L'archi-christologie comme sacrement est, en tant que pratique religieuse, l'actualisation phénoménologique de la religio chrétienne comme pur rapport au Christ dans l'intériorité divine d'affirmation et d'auto-donation de la vie.

En outre, l'archi-intelligibilité du logos johannique comme parole divine incarnée avant le temps évite la doctrine de la double nature propre à la christologie classique. Car si la chair signifie originellement ce pathos divin de l'auto-étreinte au sein duquel s'accueillent réciproquement Dieu comme Père et son Fils dans l'ipséité du premier vivant et échangent, comme «personnes », leur divinité, alors cette ipséité est également ce mode de phénoménalité éternellement passible dans laquelle est né Jésus de Nazareth en tant qu'homme visible. Si l'intériorité réciproque du Père et du Fils en Dieu présuppose une archi-chair de leur échange qui représente l'ipséité du Verbe en tant que réalisation de l'auto-révélation divine, alors le Jésus historique - comme tout autre vivant de ce monde - devient une chair individuée humainement dans l'archi-chair comme Fils de Dieu, et ceci en raison du fait que notre naissance transcendantale, cette liaison entre vie et chair dans chaque Soi, implique la condition d'un tel auto-développement, c'est-à-dire la venue-en-soi grâce à l'ipséité de la parole divine. Jésus Christ est une chair comme la nôtre en est une, capable d'éprouver la joie et la tristesse, mais il est en même temps la chair éternelle de la réalisation de la vie en Dieu. Aussi est-il le salut de l'homme comme sa « sanctification» - ce que les premiers pères de l'église interprétaient comme " divinisation $»^{2}$ : parce qu'aucun homme ne peut s'auto-fonder de soi-même en sa chair, seul le peut le Christ dans son archi-chair en tant qu'elle répète l'intériorité divine comme rapport entre Père et Fils au stade de son incarnation, et dans la mesure où elle permet également l'incarnation «au commencement» dans le devenirhumain historique.

L'incarnation éternelle ou temporelle dans le sens de l'intériorité réciproque entre Père et Fils telle que la décrit Jean dans son origine et son œuvre absolue déploie ainsi ses conséquences phénoménologiques, l'unité de l'auto-génération de la vie et de l'individuation charnelle comme concrétion de ce processus incessant, et ceci

\footnotetext{
${ }^{1}$ Pour plus de détails, cf. R. Kühn, Gabe als Leib in Christentum und Phänomenologie, op. cit., p.133 sqq., et aussi A. Vidalin, Actes du Christ et actes de l'homme. La théologie morale à l'épreuve de la phénoménologie de la vie, Paris, Parole et Silence 2012, p. 371 sqq., sur l'Eucharistie.

${ }^{2}$ Cf. Jean 17,15 sqq.
} 
jusque dans toute analyse de la chair, dont le monde n'est finalement pas à l'abri. Avec Michel Henry, nous retrouvons donc bien le cogito chrétien ou charnel, tel qu'il est d'abord développé dans la critique du gnosticisme par Irénée de Lyon ${ }^{1}$, et qui fait valoir que le corps humain n'est pas seulement susceptible de la vie divine en tant qu'il l'a reçu et le porte en soi, mais bien plus, qu'il échappe ainsi à toute ontologie mondaine matérielle. Nous l'avions plus haut désigné comme la « dignité » unique de l'incarnation entendu au sens d'une individuation transcendantale et concrète ; il nous faut maintenant souligner que le pathos de la chair, dans sa passibilité pure ou originelle, possède ce qu'aucun monde ou intentionnalité ne possèdent - la capacité de fonder quelque manifestation que ce soit dans son automanifestation affective ou pathétique. Ni la mémoire pure chez Saint Augustin, ni l'intuition conceptuelle chez Kant, ni la transcendance temporelle chez Heidegger - et pas davantage son Ereignis - n'arrivent par exemple à fonder un étant quelconque dans sa donation sensuelle originaire puisque mémoire, concept ou temps ne constituent qu'une première ouverture et une place disponible pour la manifestation du Da-sein et de l'onta, qui requièrent pour leur part, dans leur déterminité vivante comme donation concrète, d'abord une archi-phénoménalité telle qu'elle se réalise dans l'(archi-)chair. Car ce n'est que de cette manière-là que la manifestation phénoménologique devient une auto-manifestation dans laquelle toute manifestation est donnée comme manifestation à soi-même avant d'être «manifestation de quelque chose ».

Pour conclure, la réception du prologue de Jean contient une certaine pertinence gnoséologique, pour autant que le cogito charnel lie la «chair» intradivine à la « chair» humaine, et de telle sorte que tout effort subjectif transcendantal, qu'il s'agisse de la sensation ou de l'intentionnalité mondaine, recourt à une incarnation originelle en nous, qui constitue non seulement la possibilité des efforts charnels et intellectuels, mais qui reste aussi profondément liée à l'archi-chair du Christ comme commencement absolu de toute manifestation dans son auto-apparition pathétique. La vie divine vient-en-soi dans cette archi-chair, et dans cet «auto-enlacement» comme ipséisation, et toute puissance comme pouvoir vivant vient en soi de sorte que le cogito charnel touche à l'incarnation du Christ concrètement présupposée dans l'auto-épreuve passible de tout acte fondé affectivement. ${ }^{2} \mathrm{Ce}$ cogito à la fois chrétien et charnel ne contient alors pas seulement le salut de tous les individus humains dans la mesure où est à l'œuvre en eux cette incarnation originelle comme intériorité naissante divine - comme « sanctification » et « divinisation» -, mais c'est aussi le monde qui est tenu par cette unité des pratiques «christologique» et « subjective ». Car phénoménologiquement, cette pratique est, en dernière instance,

${ }^{1}$ Cf. Incarnation, op. cit., p. 180 sqq. ; M. Henry, « L'Incarnation dans une phénoménologie radicale », dans Phénoménologie de la vie, t. IV : op. cit., p. 145-154.

${ }^{2}$ Sur ce « Je peux » charnel, cf. également Incarnation, p. 195 sqq., en tant que résultat du cogito chrétien ou charnel chez Irénée et Maine de Biran. 
le corrélat purement pratique de notre agir qui en tant que pouvoir vivant est finalement lié à la possibilisation de tout Soi agissant par l'ipséisation en Christ. ${ }^{1}$

Ainsi, l'intelligibilité johannique déploie, et comme son principe constant, cette coexistence archi-originelle ou cette co-appartenance entre Dieu et Homme qui est plus qu'un rapport simplement constitué ou opaque : il s'agit plutôt d'une inhérence ou intégration de toute réalité en un principe d'une vivification indestructible à l'œuvre dans toute vie ou être dans la mesure même où il fonde la puissance d'activité de tout mouvement comme auto-mouvement nécessairement immanent. Le prologue de Jean, tel qu'il est compris par Henry, ne constitue pas seulement, dès lors, un nouveau paradigme pour une phénoménologie adéquate du monde et de la vie; il inclut aussi une "connaissance », cette archi-connaissance qui précède, en tant qu'archi-intelligibilité, chaque connaissance singulière ou régionale, et qui y est effective en tant que, charnelle et «incarnationnelle», elle est archi-affection: « Tout fut par [le Verbe] et rien de ce qui fut ne fut sans lui. » ${ }^{2}$

\footnotetext{
${ }^{1}$ Pour une « intersubjectivité » connectée au cadre du « corps mystique du Christ », cf. M. Henry, C'est moi la vérité, op. cit., p. 309 sqq. ; «L'expérience d'autrui : phénoménologie et théologie », dans Phénoménologie de la vie, t. IV, op. cit., p. 155-164.

${ }^{2}$ Jean 1,3 .
} 